\title{
Effects of bariatric surgery on cardiovascular risk factors among morbidly obese patients
}

\author{
Piotr Major', Aleksandra Kowalczuk ${ }^{2}$, Michał Wysocki², Sonia Osadnik², Michał Pędziwiatr', Anna Cłuszewska², \\ Magdalena Pisarska', Piotr Małczak', Anna Lasek', Michał Kisielewski', Andrzej Budzyński' \\ '2'nd Department of Ceneral Surgery, Jagiellonian University Medical College, Kraków, Poland \\ ${ }^{2}$ Students' Scientific Group at 2'nd Department of Surgery, Jagiellonian University Medical College, Kraków, Poland \\ ${ }^{3}$ Department of Internal Medicine and Cerontology, Jagiellonian University Medical College, Kraków, Poland
}

Article history: Received: 02.10.2016 Accepted: 16.01.2017 Published: 28.02.2017

ABSTRACT: $\quad$ Aim of the study: The aim of this study was to evaluate the influence of laparoscopic sleeve gastrectomy and laparoscopic Roux-en-Y gastric by-pass on risk factors of cardiovascular diseases.

Material and methods: We analyzed prospectively collected data of patients operated for morbid obesity who were qualified for laparoscopic sleeve gastrectomy (LSC) or laparoscopic Roux-en-Y gastric by-pass (LRYCB). Risk factors for cardiovascular diseases were assessed with the SCORE scale and both full and hard Framingham cardiovascular risk scores (FCRs). The data were collected on admission and one year after the procedures. We enrolled 264 patients (119 females, 116 males, 40.2 \pm 9.9 years old), of whom 117 underwent LRYCB and 118 LSC, respectively.

Results: Preoperatively, $12 \%$ of patients were in the high-risk category of the SCORE scale, $65 \%$ were in the moderate risk category, and $24 \%$ were in the low-risk category. The median score of the SCORE scale was 1 (1-2). Lipid-based full FCR was $34.5 \%$ (24\%-68\%) and the hard FCR was $17.5 \%$ (10\%-52\%), while the respective BMI-based FCRs were $59 \%(31 \%-84 \%)$ and $37 \%$ (15\%-67\%). One year after the procedures, the mean \%EBMIL $(62.88 \% \pm 20.02 \%)$ and \%EWL $(53.18 \% \pm 15.87)$ were comparable between both procedures. Hypertension treatment was not necessary in 33 patients after LSC and in 55 after LRYCB. Diabetes mellitus remitted in 9 and 29 patients, respectively. Both procedures significantly reduced high and moderate risk prevalence in the SCORE scale in favor of the low risk category. Surgical interventions resulted in significant reductions of FCRs 1 year after surgery $(p<0.001)$.

Conclusions: Both LSC and LRYCB lead to a significant and comparable body mass reduction. Both procedures significantly decrease of the risk of cardiovascular diseases, based on SCORE and Framingham scales.

KEYWORDS: $\quad$ morbid obesity, bariatric surgery, laparoscopic sleeve gastrectomy, laparoscopic Roux-en-Y Gastric Bypass cardiovascular risk factors, SCORE, Framingham

\section{INTRODUCTION}

Currently, the prevalence of overweight or obesity in Europe ranges between $40-50 \%$, while in the US it reaches $67 \%$ [1]. Increased body weight causes numerous comorbidities including cardiovascular diseases (CVD), hypertension, dyslipidemia, and type 2 diabetes mellitus. Obesity is associated with increased cardiovascular (CV) morbidity and mortality [2]. The risk of coronary heart disease is three times greater in obese patients compared with people with normal BMI $[3,4]$. According to WHO, in 2015, CVDs were the main cause of mortality worldwide. Approximately 17.5 million people died from CVDs in 2012, which represents almost $30 \%$ of all global deaths. In Poland, in 2010, 456 deaths per
100,000 people were due to CVDs, representing $45 \%$ of all mortality causes, while in the US, in 2007, this figure exceeded $30 \%$ [5].

Conservative methods of treating obesity do not reduce the occurrence of CV events [6,7]. Bariatric surgery is superior to conventional weight loss therapy, resulting in a long-term reduction of BMI and amelioration of metabolic and functional CVD risk factors in morbidly obese people [8]. The prevalence of obesity-related comorbidities decreases with the reduction of BMI. Such positive effects include improved insulin resistance and lipid metabolism, reduction of hypertension, amelioration of endothelial dysfunction and inflammation, reduction of ventricular hypertrophy and sympathetic overactivity [2]. 


\section{AIM OF THE STUDY}

The aim of this study was to evaluate the influence of laparoscopic sleeve gastrectomy and laparoscopic Roux-en-Y gastric by-pass on risk factors of cardiovascular diseases.

\section{METHOD}

Prospectively collected data of patients operated for morbid obesity in the $2^{\text {nd }}$ Department of Surgery, JUMC were analyzed. Guidelines of the Metabolic and Bariatric Surgery Section of the Polish Surgical Society were used as criteria for surgical treatment, i.e., body mass index (BMI) $\geq 35 \mathrm{~kg} / \mathrm{m}^{2}$ with obesity comorbidities, or BMI $\geq 40 \mathrm{~kg} / \mathrm{m}^{2}$ with or without comorbidities $[9,10]$. Patients were assessed preoperatively and allocated to laparoscopic sleeve gastrectomy (LSG) and laparoscopic Roux-en-Y gastric by-pass (LRYGB). In the absence of uniform recommendations for qualification to individual bariatric procedures, the choice of procedure was influenced by the expected impact on improvement in metabolic control or remission of diabetes, degree of excess weight loss and its sustainability. In selecting the method of treatment, we took into account the experience of our center (LSG, LRYGB), patients' BMI, age, patients' condition, obesity-related co-morbidities (especially type 2 diabetes), changes in the anatomy of the digestive tract due to disease or previous surgery (e.g. hiatal hernia, resections of the stomach, intestines), eating habits, expectations, patients' preferences, and patients' compliance in the perioperative period and during long-term care[9-12].

The study was designed to assess the influence of laparoscopic sleeve gastrectomy and laparoscopic Roux-en-Y gastric by-pass on risk factors of cardiovascular diseases, using the SCORE scale and Framingham cardiovascular risk score (FCRs), both assessed preoperatively and 12 months after surgery.The SCORE scale (systemic coronary risk evaluation) designed for the European population classifies patients into 3 risk groups (low, moderate, high), using the following parameters: age, gender, systolic blood pressure (at first ambulatory visit), total cholesterol level, and tobacco smoking. The SCORE calculator, compatible with the guidelines of the European Society of Cardiology guidelines, was used to calculate risk scores for all patients with the same parameters before and after surgery.

The 30-year risk score for cardiovascular disease was calculated using Framingham risk score for prediction of cardiovascular diseases, both BMI-associated and blood lipids-associated scales were used. The Framingham scale enables to calculate the risk of hard cardiovascular diseases and the full risk of cardiovascular diseases. Hard CVDs include coronary death, myocardial infarction, and fatal or non-fatal stroke. The full CVDs include both hard CVDs and other CVDs such as coronary insufficiency, angina pectoris, transient ischemic attack, intermittent claudication, and congestive heart failure. For calculation of BMI-associated hard and full CVD risks, we used the following qualitative and quantitative data: gender, age, systolic blood pressure, tobacco smoking, treatment of hypertension, BMI, and presence of diabetes mellitus. For calculation of lipid-based hard and full CVD risks we used gender, age, systolic blood pressure, total cholesterol serum level, HDL serum level, tobacco smoking, treatment of hypertension, and presence diabetes mellitus.

The data were collected on admission and during follow-up visits that took place one year after surgery. Height and weight were measured according to a standard protocol, and the body mass index was calculated. Systolic blood pressure was measured by professional personnel according to a standard protocol for obese patients. Fasting blood specimens, total cholesterol (TCL), high-density lipoproteins (HDL), and low-density lipoproteins levels (LDL) were measured using standardized methods. During follow-up visits (12 months after surgery), bariatric treatment effects were measured. The parameters evaluated preoperatively and on follow-up were used for calculation of percentage of excess BMI loss (\%EBMIL) and percentage of excess weight loss (\%EWL), in accordance with standard guidelines.

Statistical analysis was performed using STATISTICA 10.0 PL. The data are presented as median values with inter-quartile ranges. The chi-squared exact Fisher test, Pearson and Yates tests were used to compare qualitative data. The t-test and Mann-Whitney, Wilcoxon, and Cochrane-Cox tests analyzed quantitative differences between the groups. Statistical significance was accepted with p-values of 0.05 .

\section{ETHICS}

All procedures performed in studies involving human participants were in accordance with the ethical standards of the institutional and national research committee and with the 1964 Helsinki declaration and its later amendments or comparable ethical standards. The study was approved by the Bioethics Committee of the Jagiellonian University (KBET/156/B/2011). All patients included in the study gave written consent for participation in the study after being thoroughly informed on assumptions and aims of the study.

\section{MATERIAL}

Out of 268 patients who underwent laparoscopic bariatric procedures in the $2^{\text {nd }}$ Department of General Surgery CM UJ in 


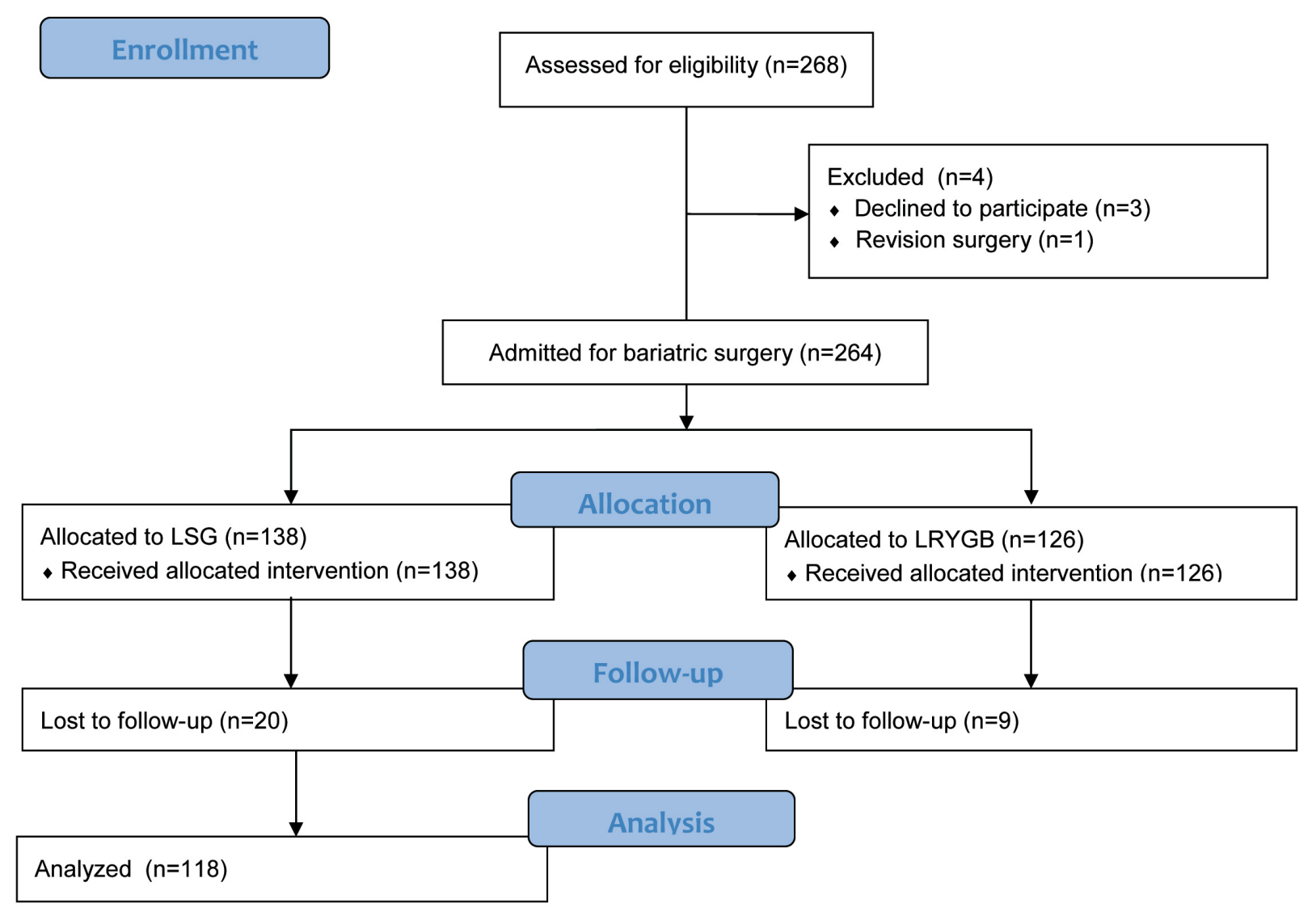

Fig. 1. Flow-chart.

2015 between January and May, 264 were enrolled in the study (119 females, 116 males, $40.2 \pm 9.9$ years). Of those, 117 (49\%) patients underwent LRYGB (58 females, 59 males, 39.3 \pm 10.6 years on average), and 118 (51\%) patients underwent LSG (61 females, 57 males, $41.1 \pm 9.3$ years on average), respectively. Table 1 presents LRYGB and LSG group characteristics. Figure 1 illustrates the flow of patients through the study.

\section{RESULTS}

Patients with type 2 diabetes mellitus were excluded from the SCORE analysis. According to the SCORE scale, preoperatively, $12 \%$ of patients were in the high cardiovascular risk category, $65 \%$ were at moderate risk, while $24 \%$ were at low risk. The median score was 1 (1-2) (Fig. 2 and 3).

Based on the lipid-related Framingham cardiovascular risk calculator, the median preoperative risk of hard CVD or coronary insufficiency, angina pectoris, transient ischemic attack, intermittent claudication, or congestive heart failure was 34.5\% (24\%-
$68 \%)$ in the entire study sample, while the median risk of coronary death, myocardial infarction, or fatal or non-fatal stroke was $17.5 \%$ (10\%-52\%). While using the BMI-related Framingham cardiovascular risk calculator, the preoperative median risks were 59\% (31\%-84\%) and 37\% (15\%-67\%), respectively (Tab. 2).

Preoperatively, type 2 diabetes mellitus and hypertension were common, more commonly present in the LRYGB group. Patients with hypertension treatment were more likely to be qualified for the LRYGB procedure. The prevalence of smoking was low. In the group of patients qualified for LSG, 6 (5\%) patients had not quit smoking preoperatively and had still smoked tobacco 1 year after the procedure. The same was observed in 14 (12\%) patients qualified for LRYGB, where all 14 patients still smoked after one year. Median total cholesterol levels were not elevated prior to surgery; however, non-optimal levels were often present, and while analyzing cholesterol fractions, dyslipidemia was commonly present (Tab. 3) .

One year after the procedures, the effects of bariatric treatment were assessed. The mean \%EBMIL was $62.88 \% \pm 20.02 \%$ in the 
entire study sample, and it was comparable in patients who underwent LSG and LRYGB $(65.5 \% \pm 21.34 \%$ vs. $60.26 \% \pm 18.88 \%$, $\mathrm{p}=0.045)$. The mean $\% E W L$ was $53.18 \% \pm 15.87$ in all patients, and it was also comparable after both procedures (LSG $54.01 \% \pm 16.79 \%$ vs. LRYGB $52.36 \% \pm 15.36 \%$, $\mathrm{p}=0.77$ ).

One year after surgery, the treatment of hypertension was not necessary in 33 patients in the LSG group and in 55 patients in the LRYGB group, while remission of diabetes mellitus was observed in 9 and 29 patients, respectively. There were no significant changes in total cholesterol levels in both groups (LSG $\mathrm{p}=0.263$, LRYGB $\mathrm{p}=0.379$ ). The average HDL level increased significantly in both groups (LSG $\mathrm{p}=0.001$, LRYGB $\mathrm{p}=0.001$ ). The median LDL level did not change significantly in the examined time period in both groups ( $\mathrm{LSG} \mathrm{p}=0.552$, $\mathrm{LRYGB} \mathrm{p}=0.394$ ). The median triglyceride levels decreased significantly one year after LSG and LRYGB ( $\mathrm{p}=0.003$ and 0.001, respectively) (Tab. 3).

Both procedures significantly reduced high and moderate SCORE risk in favor of the low risk (Fig. 2). Most of LRYGB patients had a preoperative moderate SCORE cardiovascular risk, and this was similar 1 year after the procedure (Fig. 3.). The efficacy of lowering moderate risk after one year was similar in patients undergoing the LSG procedure, and high SCORE risk in all LRYGB patients was reduced. The risk of patients who underwent LSG resembled the normal distribution, and a decrease of high and moderate SCORE risks was observed as in the case of the whole study sample.

In the analysis of the SCORE scale in both groups, we excluded 8 patients with a preoperative SCORE risk of zero. The median risk reduction on the SCORE scale was 1 point $(0.5,1 ; \mathrm{p}<0.001)$. The medians of reductions in risk scores did not differ between the procedures $(\mathrm{p}=0.24)$ and were the same as in the entire sample.

In all cases and both groups, surgical intervention resulted in a significant reduction of lipid- and BMI-related Framingham cardiovascular risks scores 1 year after the procedures $(\mathrm{p}<0.001)$. Statistical analysis showed significant differences between LSG and LRYGB in favor of a greater reduction following LRYGB; however, prior to the procedure, higher Framingham cardiovascular risks were found in patients undergoing LRYGB, which definitely contributed to a greater risk reduction (Tab. 2).

\section{DISCUSSION}

Morbid obesity is a disease that affects an increasing number of people, and it indisputably requires intervention. This is reflected by an increasing trend in the total number of bariatric procedures and bariatric surgeons in Poland [13]. Obesity is

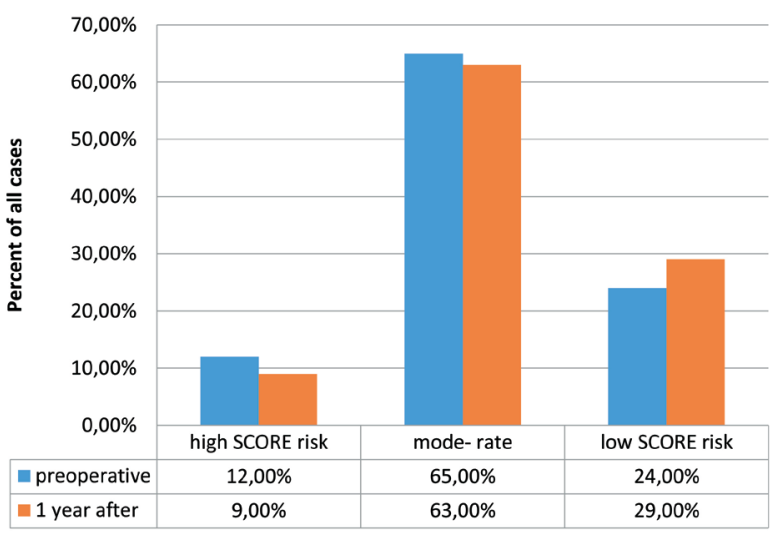

Fig. 2. SCORE risk categories preoperatively and one year after procedures.

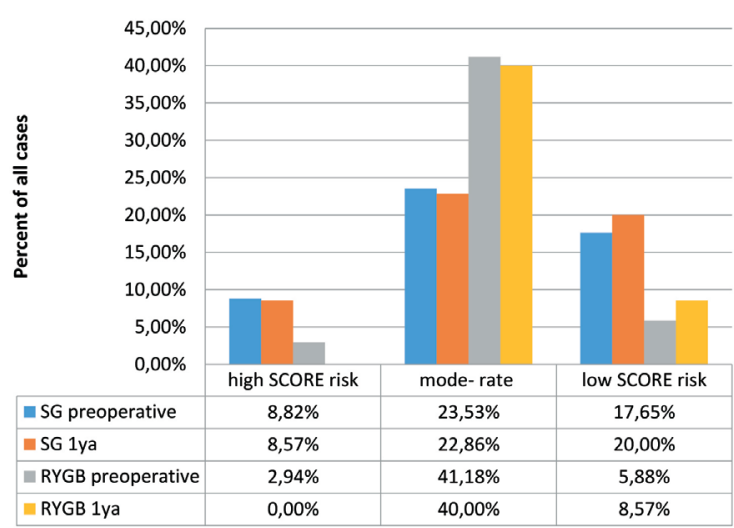

Fig. 3. SCORE risk categories preoperatively and one year after procedures in patients undergoing LSG and LRYCB.

an important cause of CVDs, and it can intensify certain CVD risk factors, for instance, dyslipidemia, hypertension, or diabetes mellitus $[5,14]$.

It has been proven by randomized clinical trials and meta-analyses that surgical intervention leads to a substantial reduction of weight and improvements in comorbidities, both of which are maintained for a long time [15-21]. The reduction of CV diseases is achieved due to the pleiotropic effects of BMI decrease after surgery, which include improvement in such comorbidities as systemic hypertension, dyslipidemia, diabetes mellitus, cardiovascular dysfunction, liver disease, and obstructive sleep apnea [2].

According to the review by Benraoune and Litwin, a significant reduction of systolic blood pressure resulting from bariatric procedures ranges from $4 \mathrm{mmHg}$ to $15 \mathrm{mmHg}$ after $1-2$ years. A complete resolution of hypertension (normal blood pressure 
Tab. I. Characteristics of patients

\begin{tabular}{|c|c|c|c|c|}
\hline & & PROCEDURE & & \\
\hline & & LSC & LRYCB & P-VALUE \\
\hline Age & years, mean $\pm S D$ & $39.33 \pm 10.56$ & $41.12 \pm 9.29$ & 0.600 \\
\hline Sex & no., total \% & $\begin{array}{l}57 \mathrm{M}(39 \%) \\
61 \mathrm{~F}(71 \%)\end{array}$ & $\begin{array}{l}59 \mathrm{M}(53 \%) \\
58 \mathrm{~F}(47 \%)\end{array}$ & 0.510 \\
\hline Height & $\mathrm{m}, \mathrm{mean} \pm \mathrm{SD}$ & $1.71 \pm 0.09$ & $1.74 \pm 0.09$ & 0.340 \\
\hline Preoperative BMI & $\mathrm{kg} / \mathrm{m}^{2}$, mean $\pm \mathrm{SD}$ & $44.74 \pm 4.52$ & $50.43 \pm 6.40$ & 0.004 \\
\hline Preoperative weight & $\mathrm{kg}$, mean $\pm \mathrm{SD}$ & $132.14 \pm 21.96$ & $154.16 \pm 27.76$ & 0.013 \\
\hline Tobacco smoking & no., total \% & $6(5 \%)$ & $14(12 \%)$ & 0.957 \\
\hline Hypertension treatment & no., total \% & $59(50 \%)$ & $96(82 \%)$ & 0.097 \\
\hline Diabetes mellitus & no., total \% & $13(11 \%)$ & $33(29 \%)$ & 0.063 \\
\hline
\end{tabular}

Tab. II. Median CV risks (IQR) according to Framingham CV risk calculator

\begin{tabular}{|c|c|c|c|c|}
\hline & OVERALL & LSC & LRYGB & $\begin{array}{l}\text { P-VALUE OF GROUPS' RISK } \\
\text { REDUCTION DIFFERENCES }\end{array}$ \\
\hline \multicolumn{5}{|c|}{ Lipid-based full Framingham CV risk } \\
\hline preoperative & $34.5 \%(24-68)$ & $25 \%(11-58)$ & $43 \%(33-69)$ & 0.001 \\
\hline 1 year after & $15.5 \%(9-27)$ & $12 \%(7-42)$ & $18 \%(12-24)$ & \\
\hline$P$-value of repeated measures & $<0.001$ & $<0.001$ & $<0.001$ & \\
\hline \multicolumn{5}{|c|}{ Lipid-based hard Framingham CV risk } \\
\hline preoperative & $17.5 \%(10-52)$ & $12 \%(4-52)$ & $22 \%(14-52)$ & 0.017 \\
\hline 1 year after & $8 \%(4-16)$ & $6 \%(3-23)$ & $9 \%(6-13)$ & \\
\hline P-value of repeated measures & $<0.001$ & $<0.001$ & $<0.001$ & \\
\hline \multicolumn{5}{|c|}{ BMI-based full Framingham CV risk } \\
\hline preoperative & $59 \%(31-84)$ & $33 \%(19-82)$ & $71 \%(59-84)$ & $<0.001$ \\
\hline 1 year after & $23.5 \%(11-57)$ & $16 \%(9-57)$ & $29 \%(20-53)$ & \\
\hline$P$-value of repeated measures & $<0.001$ & $<0.001$ & $<0.001$ & \\
\hline \multicolumn{5}{|c|}{ BMI-based hard Framingham CV risk } \\
\hline preoperative & $37 \%(15-67)$ & $18 \%(8-67)$ & $53 \%(37-66)$ & 0.002 \\
\hline 1 year after & $14 \%(5-32)$ & $8 \%(4-32)$ & $18 \%(10-28)$ & \\
\hline$P$-value of repeated measures & $<0.001$ & $<0.001$ & $<0.001$ & \\
\hline
\end{tabular}

without drug use) occurs in $20 \%-40 \%$ of patients 2 years after bariatric surgery $[21,22]$. In the Swedish Obesity Study (SOS study), the most pronounced effect on blood pressure was noted on the 2-year follow-up; however, this improvement was lost on the 10-year follow-up [21]. The improvement of dyslipidemia is frequently comparable or even larger than fallowing pharmacological treatment. LDL cholesterol levels decline ( -5 to -40 $\mathrm{mg} / \mathrm{dl})$, triglycerides also noticeably decrease $(-50$ to $-100 \mathrm{mg} /$ $\mathrm{dl})$, whereas HDL cholesterol levels typically increase (+5 to + $15 \mathrm{mg} / \mathrm{dl})$. Up to $80 \%$ of patients suffering from type 2 diabetes mellitus demonstrate a complete resolution of the disease after 1-2 years (normal fasting glucose without drug use) [21, 23]. On the 10 -year follow-up, $36 \%$ of those patients continued to be free from diabetes [21].
In our study, we noted similar results, since hypertension resolved in $56 \%$ of patients after LSG and in $57 \%$ of patients after LRYGB (overall, $57 \%$ of subjects with preoperative hypertension experienced resolution of the disease following surgery). Improvements in the lipid profile were noted in both groups. In the LSG group, 75\% of patients suffering from diabetes preoperatively did not need any medication one year later, and this was also observed in $90 \%$ of patients in the LRYGB group ( $86 \%$ of all participants). Bariatric surgery dramatically improves diabetes and insulin resistance. These changes occur within days after the procedure and therefore the enhanced glycemic regulation cannot be explained by weight loss [24]. Such findings are considered as evidence that surgical treatment, particularly gastric bypass or the sleeve procedure, provoke modifications 
Tab. III. Patients' clinical characteristics

\begin{tabular}{|c|c|c|c|c|}
\hline PROCEDURE & & LSC & LRYGB & P-VALUE \\
\hline Systolic blood pressure & mmHg, median & & & \\
\hline preoperative & & 120 & 160 & 0.023 \\
\hline 1 year after procedure & & 120 & 120 & 0.410 \\
\hline Total cholesterol level & $\mathrm{mg} / \mathrm{dl}, \mathrm{mean} \pm \mathrm{SD}$ & & & \\
\hline preoperative & & $186.07 \pm 38.84$ & $159.23 \pm 29.14$ & 0.030 \\
\hline 1 year after procedure & & $195.71 \pm 33.27$ & $146.70 \pm 21.11$ & 0.0004 \\
\hline HDL level & $\mathrm{mg} / \mathrm{dl}, \mathrm{mean} \pm \mathrm{SD}$ & & & \\
\hline preoperative & & $41.83 \pm 10.98$ & $38.03 \pm 9.89$ & 0.297 \\
\hline 1 year after procedure & & $66.42 \pm 16.44$ & $53.00 \pm 11.07$ & 0.009 \\
\hline LDL level & $\mathrm{mg} / \mathrm{dl}, \mathrm{mean} \pm \mathrm{SD}$ & & & \\
\hline preoperative & & $116.46 \pm 27.71$ & $90.08 \pm 25.64$ & 0.007 \\
\hline 1 year after procedure & & $111.69 \pm 36.20$ & $82.80 \pm 30.60$ & 0.017 \\
\hline TCC & mg/dl, median (quartiles) & & & \\
\hline preoperative & & $132.75(83.19-156.65)$ & $141.60(111.51-194.70)$ & 0.361 \\
\hline 1 year after procedure & & $82.31(61.95-133.64)$ & $82.31(64.61-108.86)$ & 0.520 \\
\hline Tobacco smoking & no., total \% & & & \\
\hline preoperative & & $6(5 \%)$ & $14(12 \%)$ & 0.957 \\
\hline 1 year after procedure & & $6(5 \%)$ & $14(12 \%)$ & 0.957 \\
\hline Hypertension treatment & no., total \% & & & \\
\hline preoperative & & $59(50 \%)$ & $96(82 \%)$ & 0.097 \\
\hline 1 year after procedure & & $26(22 \%)$ & $41(35 \%)$ & 0.63 \\
\hline Diabetes mellitus & no., total \% & & & \\
\hline preoperative & & $13(11 \%)$ & $33(29 \%)$ & 0.063 \\
\hline 1 year after procedure & & $3(3 \%)$ & $3(3 \%)$ & 0.492 \\
\hline MPV & $\mathrm{fL}, \mathrm{mean} \pm \mathrm{SD}$ & & & \\
\hline preoperative & & $10.80 \pm 0.92$ & $10.86 \pm 0.95$ & 0.854 \\
\hline 1 year after procedure & & $10.62 \pm 0.97$ & $11.16 \pm 1.16$ & 0.156 \\
\hline 1 year after procedure BMI & kg/m2, mean $\pm S D$ & $31.93 \pm 4.50$ & $35.96 \pm 6.69$ & 0.047 \\
\hline 1 year after procedure weight & kg, mean $\pm S D$ & $93.31 \pm 18.41$ & $110.23 \pm 26.80$ & 0.041 \\
\hline
\end{tabular}

in the gut-secreted hormones that sharply regulate metabolism [24]. Despite the lack of smoking cessation in 20 patients, we decided not to exclude them from the analysis; however, this could slightly increase the risk for cardiovascular events. Recent prospective, case-control, population studies have also reported surgical treatment to prolong life expectancy in the morbidly obese. Adams et al. reported that bariatric surgery was responsible for a $56 \%$ reduction in mortality caused by coronary heart disease and a $40 \%$ decrease in all cause fatality (retrospective cohort study, 7.1 years of follow-up) [25]. Christou et al. demonstrated that LRYGB was associated with an $82 \%$ risk reduction for cardiovascular morbidity and an $89 \%$ risk reduction for overall mortality (5 years follow-up) [26]. The results of the SOS study supported this finding by demonstrating a reduction of both fatal and total cardiovascular events, by $53 \%$ and $33 \%$, in a group of patients after surgery compared to controls, respectively [27].
We proved that both procedures significantly reduced high and moderate SCORE risk in favor of the low risk; however, patients with type 2 diabetes mellitus were excluded from the SCORE analysis, because this scale is not recommended for cardiovascular risk assessment in patients with this comorbidity. Patients with type 2 diabetes mellitus are already at a higher risk than indicated by the SCORE scale. We chose the Framingham cardiovascular risk scores (FCRs) to estimate the 30-year CVD risk change in all individuals, and we revealed a relative risk reduction by more than a half (50-66\%, depending on the used model, lipid or BMI, and type of surgery). The lipid model of FCRs seems to be the most adequate for this research, as it was not influenced by weight and because it is the optimal model to evaluate the significance of bariatric surgery in the CVD risk improvement [2]. The relative improvement of $58 \%$ was observed following LRYGB, considering all CV events (full FCRs), and we observed an improvement of 59\% 
of fatal CV events incidence (hard FCRs). For LSG, these figures were $52 \%$ and $50 \%$ respectively. In the BMI model, we observed an improvement of 59\% (full FCRs) and 66\% (hard FCRs for LRYGB); the respective values for the LSG group were $51 \%$ and $55 \%$.

Several groups have estimated the alterations in long-term risk of CV events due to beneficial effects of surgical treatment on cardiac risk factors. Based on Framingham risk scores, Vogel et al. assessed that the 10 year-risk would decrease from $6 \%$ at baseline to 3\% following LRYGB [28]. Batsis et al. showed that LRYGB reduced the prevalence of $\mathrm{CV}$ events relatively by $51 \%$ (from $37 \%$ to $18 \%$ ) and the prevalence of cardiovascular death by $55 \%$ (from $5.8 \%$ to $2.6 \%$ ) [29].

Gastric by-pass is more frequently studied, while there are limited data showing the influence of other bariatric procedures on FCRs. The study by Largent et al. analyzed patients after the laparoscopic adjustable gastric banding procedure, showing a reduction of estimated risk for CVD by 33\% [30]. This study revealed a slight predominance of LRYGB over LSG in reducing the CVD risk. However, this can be due to a limited number of participants and differences in patients' characteristics between the groups.

A limitation of this study is the use of the Framingham CVD risk scale in the Polish population. The authors of the Framingham CVD risk assessment model suggest to limit its application only to the population of African-Americans and Americans of European descent. However, the Polish Cardiac

\section{REFERENCES}

1. Nguyen, D.M. and H.B. El-Serag, The epidemiology of obesity. Gastroenterol Clin North Am, 2010. 39(1): p. 1-7.

2. Boido, A., et al., Bariatric surgery and prevention of cardiovascular events and mortality in morbid obesity: mechanisms of action and choice of surgery. Nutr Metab Cardiovasc Dis, 2015. 25(5): p. 437-43.

3. Calle, E.E., et al., Body-mass index and mortality in a prospective cohort of U.S. adults. N Engl J Med, 1999. 341(15): p. 1097-105.

4. Tavani, A., et al., Body weight and risk of nonfatal acute myocardial infarction among women: a case-control study from northern Italy. Prev Med, 1997. 26(4): p. 550-5.

5. Roger, V.L., et al., Heart disease and stroke statistics--2011 update: a report from the American Heart Association. Circulation, 2011. 123(4) p. e18-e209.

6. Al-Habbal, Y., C. Christophi, and V. Muralidharan, Aneurysms of the splenic artery - a review. Surgeon, 2010. 8(4): p. 223-31.

7. Trastek, V.F., et al., Splenic artery aneurysms. Surgery, 1982. 91(6): p. 694-9.

8. Ikeda, O., et al., Nonoperative management of unruptured visceral artery aneurysms: treatment by transcatheter coil embolization. J Vasc Surg, 2008. 47(6): p. 1212-9.

9. Wyleżoł, M., et al., Polskie rekomendacje w zakresie chirurgii bariatrycznej. Videosurgery and other miniinvasive techiques, 2009.4(Supl. 1): p. S31-S34.
Society and the Polish Society for Atherosclerosis Research allow the use of the Framingham model due to its simplicity and reliability in everyday medical practice [31,32]. The evaluation of the risk of CVD using this scale may be part of routine diagnosis and a reference point, when deciding on medications and the type and aggressiveness of interventions for prevention of CVD.

Based on our study, both methods, i.e., laparoscopic Roux-en-Y gastric bypass and laparoscopic sleeve gastrectomy, lead to a significant and comparable body mass reduction and a significant decrease of the risk of CVD. It seems that prevention of CVDs due to surgical treatment is more strongly associated with the initial status of patients than the type of surgery. Moreover, the almost universal improvement in CVD risk, independent of baseline BMI or surgery method, suggests that bariatric surgery may be a reasonable solution to reduce CVD risk in individuals with primary BMI below the prevailing standards. Large prospective studies are needed to assess this issue in morbidly obese patients [2].

\section{CONCLUSION}

Both laparoscopic sleeve gastrectomy and laparoscopic Rouxen-Y gastric bypass lead to a significant and comparable body mass reduction, and they both significantly decrease the risk of cardiovascular diseases, based on the SCORE scale and Framingham scores.

10. Budzyński, A., et al., Polskie rekomendacje w zakresie chirurgii bariatrycznej i metabolicznej. Medycyna Praktyczna - Chirurgia, 2016. 6.

11. Kaska, $Ł$., et al., A prospective evaluation of the influence of three bariatric procedures on insulin resistance improvement. Should the extent of undiluted bile transit be considered a key postoperative factor altering glucose metabolism? Wideochirurgia i inne techniki małoinwazyjne/Videosurgery and Other Miniinvasive Techniques, 2015. 10(2): p. 213-228.

12. Proczko-Markuszewska, M., et al., Early results of Roux-en-Y gastric by-pass on regulation of diabetes type 2 in patients with BMI above and below $35 \mathrm{~kg} /$ m2. Pol Przegl Chir, 2011. 83(2): p. 81-6.

13. Janik, M.R., E. Stanowski, and K. Paśnik, Present status of bariatric surgery in Poland. Wideochirurgia i inne techniki małoinwazyjne/Videosurgery and Other Miniinvasive Techniques, 2016. 11(1): p. 22-25.

14. Saw, E.C., W. Ku, and S. Ramachandra, Laparoscopic resection of a splenic artery aneurysm. J Laparoendosc Surg, 1993. 3(2): p. 167-71.

15. Carr, S.C., et al., Current management of visceral artery aneurysms. Surgery, 1996. 120(4): p. 627-33; discussion 633-4.

16. Arepally, A., et al., Treatment of splenic artery aneurysm with use of a stent-graft. J Vasc Interv Radiol, 2002. 13(6): p. 631-3.

17. Arca, M.J., et al., Splenic artery aneurysms: methods of laparoscopic repair. J Vasc Surg, 1999. 30(1): p. 184-8. 
18. Piffaretti, G., et al., Splenic artery aneurysms: postembolization syndrome and surgical complications. Am J Surg, 2007. 193(2): p. 166-70.

19. Gao, B.L., et al., Delayed coil migration from a small wide-necked aneurysm after stent-assisted embolization: case report and literature review. Neuroradiology, 2006. 48(5): p. 333-7.

20. Saltzberg, S.S., et al., Is endovascular therapy the preferred treatment for all visceral artery aneurysms? Ann Vasc Surg, 2005. 19(4): p. 507-15.

21. Guillon, R., et al., Management of splenic artery aneurysms and false aneurysms with endovascular treatment in 12 patients. Cardiovasc Intervent Radiol, 2003. 26(3): p. 256-60.

22. Agrawal, G.A., P.T. Johnson, and E.K. Fishman, Splenic artery aneurysms and pseudoaneurysms: clinical distinctions and CT appearances. AJR Am J Roentgenol, 2007. 188(4): p. 992-9.

23. Yamamoto, S., et al., Transcatheter coil embolization of splenic artery aneurysm. Cardiovasc Intervent Radiol, 2008. 31(3): p. 527-34.

24. Kagaya, H., et al., Long-term results of endovascular treatment for splenic artery aneurysms. Int Angiol, 2011. 30(4): p. 359-65.

25. Adams, T.D., et al., Long-term mortality after gastric bypass surgery. $\mathrm{N}$ Engl J Med, 2007. 357(8): p. 753-61.
26. Moriyama, H., et al., Laparoscopic approaches for splenic artery aneurysms. Minim Invasive Ther Allied Technol, 2012. 21(5): p. 362-5.

27. Trastek, V.F., P.C. Pairolero, and P.E. Bernatz, Splenic artery aneurysms. World J Surg, 1985. 9(3): p. 378-83.

28. Lakin, R.O., et al., The contemporary management of splenic artery aneurysms. J Vasc Surg, 2011. 53(4): p. 958-64; discussion 965.

29. Mandal, S., P.M. Das, and A.K. Mandal, Caroli's syndrome leading to splenic artery aneurysm: a rare presentation. Int J Surg Pathol, 2009. 17(1): p. 78-80.

30. Largent, J.A., et al., Reduction in Framingham risk of cardiovascular disease in obese patients undergoing laparoscopic adjustable gastric banding. Adv Ther, 2013. 30(7): p. 684-96.

31. International Task Force for Prevention of Coronary Heart Desease / International Atherosclerosis Society, Skala Framingham, in Zapobieganie Chorobie Niedokrwiennej Serca Poradnik Kieszonkowy. 2003, Verso s.c.: Szczecin. p. 22-26.

32. Hrynkiewicz-Szymańska, A., Diagnostyka choroby niedokrwiennej serca w grupie pacjentówz cukrzyca. Kardiologia na co Dzień, 2010. 5: p. 45-47.

\begin{tabular}{llll}
\hline Word count: $3100 \quad$ Page count: 9 & Tables: $3 \quad$ Figures: $3 \quad$ References: 32 \\
\hline
\end{tabular}

DOI: $\quad 10.5604 / 01.3001 .0009 .7176$

Table of content: http://ppch.pl/resources/html/articlesList?issueld=9609

Copyright: Copyright @ 2017 Fundacja Polski Przegląd Chirurgiczny. Published by Index Copernicus Sp. z o. o. All rights reserved.

Funding: The publication of this article was supported by the Faculty of Medicine, Jagiellonian University Medical College, Leading National Research Centre (KNOW) 2012-2017

Competing interests: The authors declare that they have no competing interests.

The content of the journal „Polish Journal of Surgery" is circulated on the basis

- of the Open Access which means free and limitless access to scientific data.

This material is available under the Creative Commons - Attribution 4.0 GB. The full terms of this license are available on: http://creativecommons.org/licenses/by-nc-sa/4.0/legalcode

Corresponding author: Piotr Major, Il Katedra Chirurgii Ogólnej - Uniwersytet Jagielloński Collegium Medicum, Kraków, Polska; e-mail: majorpiotr@gmail.com

Cite this article as: Major P., Kowalczuk A., Wysocki M., Osadnik S., Pędziwiatr M., Głuszewska A., Pisarska M., Małczak P., Lasek A., Kisielewski M, Budzyński A.; Effects of bariatric surgery on cardiovascular risk factors among morbidly obese patients; Pol Przegl Chir 2017: 89 (1): 41-49 
